\title{
Femoral Neck Cortical Bone in Female and Male Hip Fracture Cases: Differential Contrasts in Cortical Width and Sub- periosteal Porosity in 112 Cases and Controls.
}

\author{
Jon Power ${ }^{1}$, Nigel Loveridge ${ }^{2}$, Heikki Kröger ${ }^{3}$, Martyn Parker ${ }^{4}$, \\ Jonathan Reeve ${ }^{5 *}$
}

${ }^{1}$ Department of Biological Sciences, University of Chester, Chester $\mathrm{CH} 1$ 4BJ, UK

${ }^{2}$ Division of Trauma and Orthopaedic Surgery, University of Cambridge, Box 180, Addenbrooke's Hospital, Cambridge, CB2 2QQ, UK

${ }^{3}$ Department of Orthopaedics, Traumatology and Handsurgery, Kuopio University Hospital, P.O.BOX 100, FIN-70029 KYS, Kuopio, FINLAND.

${ }^{4}$ Trauma and Orthopaedics, Peterborough City Hospital, Edith Cavell Campus, Bretton Gate, Peterborough PE3 9GZ, UK

${ }^{5}$ NIHR Biomedical Research Centre, Institute of Musculoskeletal Sciences, Nuffield Orthopaedic Centre, Oxford, OX3 7LD, UK

* Author for Correspondence: jonathan.reeve@ndorms.ox.ac.uk

Running Head: Femoral Neck Cortex and Hip Fracture 


\section{ABSTRACT}

Objectives: To quantitate differences between cases of hip fracture and controls in cortical width around the mid-femoral neck in men and women.

Methods: Over 5 years, 64 (14 male) participants over age 55 (mean 79) years, who had never taken bone-active drugs and suffered intra-capsular hip fracture treated by arthroplasty, donated their routinely discarded distal intra-capsular femoral neck bone for histomorphometry. After embedding, complete femoral neck cross sections from the cut surface near the narrowest part of the neck were stained with von Kossa and cortical width was measured radially every 5 degrees of arc. Control material ( $n=48,25$ male) was available through consented post mortems prior to the year 2000. Cortical widths were averaged for circumferential octants, each representing 45 degrees of arc. Divergence of individual cortical widths from their means was also examined.

Results: Because sections were required to have a complete cortex, sampling was biased towards cases with sub-capital versus trans-cervical fractures. Compared to sex- and age matched controls, male cases showed larger relative differences in cortical widths than female cases. Unexpectedly, cortical widths in female but not male cases also showed marked overrepresentation of extremely narrow $(<0.1 \mathrm{~mm})$ cortical widths, located mainly posteriorly. The numbers of these very narrow cortical widths observed per subject retrospectively predicted female fracture status in logistic regression independently of mean cortical width values. Together with mean cortical width differences, the numbers of measured cortical widths $<0.1 \mathrm{~mm}$ (out of 72 measured) raised the sensitivity of predicting fracture status in women from 48 to $80 \%$ at $80 \%$ specificity. In almost all cases, very narrow cortical widths were identified in regions enclosing a cortical pore roofed on its endosteal surface by thin structural bone defined a priori as trabecular.

Conclusions: Cortical widths $<0.1 \mathrm{~mm}$ probably reflect zones where endosteal cortex has been trabecularised through expansion of an un-refilled sub-endosteal canal close to the periosteum. Persistent cortical defects occurring near the periosteal surface, where mechanical loading exerts its greatest stresses, are likely to result in extremes of localized concentrations of stress during a fall, unknown in young normal fallers. Such defects have the potential to help explain the excess of hip fractures among elderly women. Prevention of sub-periosteal tunnelling by osteoclasts might explain in part the additional benefits, beyond an increase in bone density, of treatments that reduce excessive bone resorption or else stimulate new bone formation on previously resorbed surfaces.

\section{KEYWORDS}

femoral neck fracture; cortical bone thinning; sub-periosteal pores; trabecularisation of the cortex. 


\section{Introduction}

In previous work we found micro-structural abnormalities of the femoral neck in intra-capsular hip fracture cases that were most marked in cortical bone [1-5]. In the fracture cases, there was excessive cortical thinning on the supero-posterior to infero-anterior axis [2],the axis that is maximally loaded on impact from a fall. There was also increased cortical porosity [1]. The proportion of actively remodeling haversian canals was also shown to be significantly elevated in the fracture cases [6].

These results justified a three centre collaboration between Cambridge and Peterborough (UK) with Kuopio (Finland) in which intact femoral neck biopsies of bone routinely discarded at hemiarthroplasty with cortices that were un-fractured at the basi-cervical level were harvested sequentially from freely consenting, sequentially presenting men and women with hip fracture. The surgical procedure involved the careful preservation intact of the discarded distal stump of the femoral neck. [7].

All studies on the proximal femur and other sites containing cortical and trabecular bone require the definition of a boundary between these two categories of bone tissue. Here we avoid definitions based on tissue history, since osteoblastic bone formation may have occurred many years previously, and use instead a definition based on current anatomy. So if a structural element that may previously have been cortical is bounded on both sides by bone marrow or the un-calcified content of a cortical canal and is less than $0.1 \mathrm{~mm}$ thick it is defined as trabecular, with one exception. If it is bounded on one side by the periosteum it is defined as cortical bone whatever its thickness, along with the remaining elements that are not defined as trabecular.

Here we report on the macro- and micro-anatomical differences between cases and controls in cortical width around the 360 degrees of the femoral neck cross section. Our aims were threefold: first to provide an enlarged and representative comparison between cases and controls in women; second to obtain a statistically adequate sample of male cases of hip fracture for comparison with controls; and third to determine whether there were at a microscopic level beneath the resolution of current in vivo imaging differences between cases and controls that 
might allow a clearer statistical contrast to be made between cases and controls, and could also advance our basic understanding of hip fragility in the elderly. 


\section{Methods}

\section{Study Design}

This was a three-centre case:control study of white British and Finnish men and women who had suffered an intra-capsular hip fracture. We aimed to recruit at least 50 cases and 50 controls aged 50 or over, with equal numbers of male and female controls. A second statistical requirement was that the ratio of cases to controls in each sex should be within the range 1:2$2: 1$. In the event we recruited 48 controls before consent procedures changed for the controls following the UK Alder Hay enquiry, which halted their recruitment. Because of slow recruitment of male compared to female cases, recruitment of male cases was extended 18 months beyond the time-frame applying to controls and female cases.

\section{Subjects}

Living participants with a prior hip fracture (50 female, 14 male) were recruited from our three centres over $7 \frac{1}{2}$ years beginning in 1996. Each had suffered an intra-capsular fracture of the femoral neck resulting from trauma equal to or less than a fall from standing height (Table 1). Cases of intertrochanteric fracture and cases with evidence of hip joint osteo-arthritis (KellgrenLawrence Grade 3 or 4) were excluded by design. To understand the effects of our inclusion and exclusion criteria on the types of fracture studied, in the Cambridge Centre all but two of the participants had their fracture type retrospectively classified by one experienced radiologist blind to the clinical record from their routine peri-operative radiographs, as Sub-capital, Midcervical or (for the purpose of exclusion) as inter-trochanteric. The Kellgren Lawrence score of the hip joint on the fractured side, if not used already by the surgeon to exclude the participant, was confirmed as being 2 or less by the Radiologist. It was not possible for the radiographs from the other centres to be read by the same radiologist for logistical reasons and exclusions on radiological grounds were determined by the operating surgeon. An important inclusion criterion that distinguished the present from our previous study [2] was that the circumference of the cortex should not appear macroscopically to be broken either by the trauma of the clinical fracture or inadvertently during retrieval of the biopsy during the operation. This did not exclude some sections having apparent breaks in cortical continuity only on microscopic examination.

\section{Sample Collection}




\section{a) Cases}

During the fixation of an intra-capsular fracture, it is routine to replace the femoral head and femoral neck proximal to the fracture surface by a prosthesis. The prosthesis has to be fitted to the shaft of the femur after removing an appropriate length of the femoral neck distal to the fracture surface. Our biopsy procedure recovered this portion of femoral neck bone distal to the fracture surface with the intention of causing minimal further thermal and mechanical damage [7] at routine fracture fixation, which was normally scheduled within one day of admission if the patient was clinically fit. According to the Declaration of Helsinki, written informed consent to participate in the study was obtained from each participant pre-operatively, following approval by the appropriate Institutional Review Committees.

Exclusion of biopsies with incomplete femoral neck cortices, due to accidental damage during surgery or to extension of the fracture surface through the lateral aspect of the cortex, or else due to femoral head osteoarthritis resulted in an exclusion rate of $60 \%$.

\section{b) Controls (non-fractured)}

For the control material, a similar sized sample of femoral neck bone was removed at routine post mortem following the participant's relatives' consent. The control subjects were selected from routine postmortem cases in Cambridge with no previous history of disease such as carcinoma or Paget's disease affecting bone or usage of drugs such as glucocorticoids known to affect bone metabolism. In addition, subjects were not included if they had been admitted to hospital for more than 14 days prior to death or if they were admitted from other hospitals or residential care. Recruitment of Controls in the Cambridge centre was concluded prior to 2000.

\section{Sample preparation}

All biopsies were fixed in $80 \%$ ethanol and embedded in methyl-methacrylate without prior decalcification [2]. Having identified the inferior cortex (which is always the thickest cortical region), the anterior region was identified by which side (i.e., left or right) of the proximal femur the biopsy was taken. Ten-micron sections, parallel to the face of the biopsy, were cut from the trochanteric (ie basi-cervical) side of the biopsy with a Jung Polycut E microtome (Leica, Milton Keynes, U.K.) and stained using the von Kossa protocol [2]. 


\section{Image analysis}

\section{a) Periosteal and Endosteal Boundaries}

von Kossa sections (one per participant) were reviewed under the microscope for the purpose of satisfying our inclusion criteria. Since the complete cross section of a femoral neck was larger than the maximum field size, we "stitched" together the images of adjacent fields after they had been captured with a digital camera attached to a microscope (magnification $\times 10$ ). Cross sectional images were then quantitated using an Image-J based program that identified individual pixels in the image as bone (black) or not bone (white). The dimensions of each pixel were $8.10^{-3} \times 8.10^{-3} \mathrm{~mm}$ square. The program sought first to identify the periosteal boundary of the section. Since the analysis of each section required a complete periosteal boundary in those cases where it was missing (due to sectioning artefacts or the presence of the fracture surface), the broken boundary was re-connected manually by digitally painting in a line between 2 and a maximum of 4 pixels wide (based on Pythagorean geometry, the range of widths of operator-drawn re-connections therefore lay between 16 and $32^{*} \sqrt{2}$ : ie range 16$\left.46^{*} 10^{-3} \mathrm{~mm}\right)$.

To define the cortex it was then necessary to define its endosteal boundary. The underlying principle was that any strut of bone that was bounded on both sides by bone marrow or the contents of a Haversian space was part of the cancellous compartment if it was less than approximately $100^{*} 10^{-3} \mathrm{~mm}$ thick. For geometrical reasons this figure of $100^{*} 10^{-3} \mathrm{~mm}$ covered an absolute range of $80-113^{*} 10^{-3} \mathrm{~mm}$ to allow for the rectangular nature of the pixellation. To remove trabecular elements, an automatic routine was employed that eroded all bone surfaces one pixel at a time in a series of five such erosions. In this way, from Pythagorean geometry, trabecular and trabecular-like bone with two parallel surfaces would be removed in the event that it was less that $0.08 \mathrm{~mm}$ thick if its edges were parallel to the orientation of the pixels, less than $0.113 \mathrm{~mm}$ thick if its edges were orientated at $45^{\circ}$ to those of the pixels and for any other orientation somewhere between these two figures. If this erosion process reached the periosteum, the periosteal surface was preserved unbroken and allowed to retreat an appropriate distance. After completing five erosions the software then reversed the process by adding in sequence 5 pixels of imaged bone onto those surfaces that remained available. In this way bone defined as cortical was built back up to its original width. Fig 1 illustrates how the program implemented the drawing of an endosteal boundary between the cortex and the cancellous bone. 
Because the trauma of hip fracture frequently damaged the trabecular bone structure inside the cortical shell, we made no attempt to analyse the trabecular bone for its contribution to fragility.

\section{b) Locating cortical width measurements}

We then automated the selection of locations round the cortex where cortical width measurements were made. Having defined the endosteal boundary, the coordinates of the centre of area of the cross section were calculated from the means of the $x$ and $y$ coordinates respectively of all pixels located within the periosteal boundary, including those pixels defined as being located within the marrow/trabecular bone space. Having selected the most inferior point on the cortex, 72 cortical width measurements were made based on one measurement per 5 degrees of arc from the centre of area. For this purpose the software drew 72 radial lines from the centre of area and the distances between the intercept of each radial line with the two surfaces, endosteal and periosteal, was calculated from their respective coordinates. Each cortical width measurement was then allocated to one of eight octants as previously described [2]. To exclude cortical widths that might have been synthetic (ie drawn by the operator) the $4.1 \%$ of measurements $<0.045 \mathrm{~mm}$ were censored. To further exclude large cortical width measurements that might have included part of a trabecular strut connecting to the endosteal surface, all CWs > 3 SDs greater than the mean value for the octant in question, assuming a normal distribution across the whole population of biopsies, were excluded (a further $1.0 \%$ ). So as to investigate the ability of the original 72 measurements to adequately represent the variability of cortical widths within regions, a subset of biopsies was re-analysed in the same way but with 360 measurements of cortical width made at intervals of 1 degree of arc.

\section{c) Section Location}

It was not practical to specify the location of the section analysed with respect to its caudalcranial axis. Crabtree et al [5] showed that while the proportion of cortical bone in the femoral neck is dependent on location along this axis, the cross section also becomes progressively more ellipsoid with distance from the femoral head. Therefore we measured the maximum and minimum diameters of each femoral neck section, expressed them as a ratio (Max:Min ratio) and used this ratio as a covariate to adjust for between-subject differences in section locations along the caudal-cranial axis. 


\section{Analysis of Data}

\section{a) Descriptive Data}

From the original 72 uncensored and censored cortical width measurements, in each octant there were up to nine uncensored measurements of cortical width. The means and SDs of these widths were calculated for each octant. The distributions of cortical widths were then contrasted in cases and controls using Q-Q plots to explore the possibility that cases had an excess of very small cortical widths that was not reflected in the summary statistics. Differences between cases and controls are described as reductions if the cases showed lower values and increases if the differences were higher, without implying there had been changes over time.

\section{b) Multivariate statistical modeling of cortical width}

Mean cortical width values for each subject and octant were entered into linear statistical models that included: age; sex; case versus control status; position of histological section along the femoral neck as reflected in the ratio of maximum to minimum diameter of the cross section (max-min ratio); and all significant interactions between them. MANOVA was used to contrast the effects of age, sex and case-control status on the 8 octants per subject, using repeated measures.

\section{c) Prediction Models for Hip Fracture}

Structures under excess mechanical load generally initiate fracture at their weakest point where the local load is greatest. Therefore we examined whether some measure relating in some way to the local concentration of loading forces might contribute to fracture prediction. From our data, such candidate measures included the numbers of cortical widths below a pre-defined width threshold.

Logistic regression with fracture status as the outcome variable was used to determine whether measurements of mean and minimum cortical width, either in each octant or in the whole cross section, provided predictive information on fracture status. We then included numbers of cortical widths below a predetermined threshold suggested by the maximum deviation of the Q$Q$ plots for the fracture cases compared to controls. When there was a significant interaction between sex and another independent determinant, male and female data were modelled 
separately. In logistic models the area under the ROC curve was used as a measure of retrospective prediction of fracture status. Included covariables additional to sex and cortical width measurements were age, investigational centre, max:min ratio and all significant interactions between them. To simplify these prediction models, non-significant determinants ( $p>0.01$ ) were removed beginning with all interaction terms at $p>0.01$ in order of relatively increasing significance and then other terms until all remaining independent determinants were significant at $p<0.01$. 


\section{$\underline{\text { Results }}$}

\section{Fracture classification}

Among the biopsies from Cambridge, four in the sequence from sub-capital fracture cases were unavailable, being retained in the frozen state for pilot work on the study of NOS isoforms [8], while two could not be classified for fracture type due to missing radiological images. Fourteen of the remaining 24 Cambridge biopsies were classified as sub-capital. Fifty one percent of biopsies that were offered for study from sub-capital fracture cases and $14 \%$ from trans-cervical fracture cases were classified as having cortices complete around the circumference of the cut face of the biopsy and therefore were eligible for inclusion.

\section{Data censoring and cleaning}

Table 1 shows the characteristics of the studied participants. Across this population, it was necessary to censor $1 \%$ of cortical width measurements because they coincided with gaps in the cortex; an additional $0.6 \%$ of cortical width measurements were also censored because they coincided with the junction of a trabecula with the cortex creating a thickness that included an unknown proportion attributable to trabecular bone. It was more likely that a case had one or more cortical widths censored than a control (median number of widths censored: cases 1 , interquartile (IQR) range $0-5$ out of 72 ; controls median 0 , IQR $0-1 ; p<0.001$ Wilcoxon test). The distributions of participants' chronological ages and their section locations along the caudal-cranial axis of the femoral neck are also shown in Table 1. Table 2 shows the statistical effects on the mean cortical width of the patient's age, fracture status and location of the biopsy section along the femoral neck as indicated by the Max:Min ratio.

\section{Multivariate modeling of statistical determinants of mean cortical width by octant.}

The eight mean cortical width measurements per subject (8 octants, max 9 measurements per octant in each subject) were made the outcome variable in MANOVA. Independent determinants entered in the model were sex, fracture status, Max:Min ratio and age. There were no significant interactions ( $p>0.05$ ) between the independent determinants in either the between-subject or the within-subject analyses, so data for men and women were combined. 
The only between-subject determinants that were significant $(<0.05)$ for the between-subject analysis were fracture status $(p<0.0001)$ and the max-min ratio $(p<0.025)$. The within-subject analysis showed no significant determinants influencing the contrasts in mean CWs between octants. Because the residuals were not normally distributed, the MANOVA was repeated after taking logarithms of the mean cortical widths, which provided partial correction. The results were similar.

Descriptive statistics of cortical widths by octant in cases and controls.

Table 3 shows the geometric means of the cortical widths in each octant for cases and controls. Male cases showed larger relative reductions than female cases compared to their controls in all octants. It is demonstrated that the relative reductions in the cases compared to controls were highest in the infero-posterior and posterior octants in both men and women while the three superior octants showed the smallest relative reductions in the cases compared to the controls. Despite the greater reductions in men, male and female cases showed similar patterns of relative cortical width reduction compared to controls with a correlation coefficient between mean case vs control reductions of 0.87 across the eight octants.

Fig 2 shows the quantile-quantile (Q-Q) plots of the CW measurements per octant for the cases and controls (males and females shown separately). There is a noteworthy over-representation of very narrow CWs in the female cases compared to the controls, which appears almost entirely absent in the male cases. Fig 3 shows the mean number of $\mathrm{CWs}<0.1 \mathrm{~mm}$ per octant in female cases and controls as a function of the angular distance from the inferior octant.

The mean CW varied considerably across different octants because of the natural asymmetry of $\mathrm{CW}$ in the elderly femoral neck. Because fracture resistance might be related to the variability of cortical width as well as to mean cortical width, as shown in Table 4 we examined the degree to which the minimum (out of 9 measurements per octant) differed from the mean $\mathrm{CW}$ at the level of the octant. It can be seen that minimum values in an octant as proportions of mean values tended to fall in the range $0.45-0.75$ with little difference between cases and controls.. However, when comparison was made between the $10^{\text {th }}$ centiles of the ratios of minimum to mean CWs, which explored the smallest values found, larger case:control differences in these ratios emerged in the posterior and inferior regions. This reflects the tendency shown in Fig 3 for some but not all female (but not male) cases of hip fracture to have 
one or two ultra-low cortical width measurements in especially the posterior octants.

\section{Retrospective prediction of hip fracture}

In consequence we modelled the possibility that numbers of CWs $<0.1 \mathrm{~mm}$ might retrospectively "predict" hip fracture status in logistic regression analysis. In all models adjustments were made for age and the max:min ratio. Separate models were developed for men and women. The area under the Receiver Operator Characteristics (ROC) curve was used to screen models for their predictive ability, together with their sensitivity at $80 \%$ specificity (women) and 95\% specificity (men), these numbers being chosen to reflect typical lifetime hip fracture risks in the UK. In women, after adjusting for age and the max:min ratio the area under the ROC curve when the logarithm of the mean CW was used predictively was $73 \%$ and the sensitivity was $48 \%$ at $80 \%$ specificity. Adding the number of CWs $<0.1 \mathrm{~mm}$ (out of a maximum count of 72) as an additional independent determinant into the model further increased the area under the ROC curve to $82 \%$ and the sensitivity rose to $80 \%$ at $80 \%$ specificity (Fig 4 ).

In men, after adjusting for age and Max:Min ratio, mean log CW alone produced an area under the ROC curve of $70 \%$ with a sensitivity of $20 \%$ at $95 \%$ specificity. In contrast to women, no further improvement to the area under the ROC curve or the sensitivity at $95 \%$ specificity was obtained by including the number of CWs of $<0.1 \mathrm{~mm}$ width in the model (Fig 4). 


\section{Discussion}

Female cases of hip fracture differed substantially from controls in having increased numbers of very localized, microscopically thin cortical regions. These most likely reflect previous intracortical (ie haversian) or less likely endosteal resorption that was not promptly followed by adequate bone formation during the remodeling cycle. These localized cortical defects are distinct from the generalized thinning of the cortices compared to controls in hip fracture cases, which compared to females is comparatively more pronounced in male cases and has been previously documented both in- and ex- vivo. It is possible that both generalised cortical thinning and the localized defects reported here reflect previous remodeling imbalances, occurring respectively intra-cortically and at the endosteal surface, combined with possibly reduced sub-periosteal bone formation [9-17]. When examining predictors of hip fracture status in logistic regression, only in women did numbers of localized microscopic regions of excessive cortical thinning contributed substantially and independently to the correct retrospective assignment of fracture category, after adjusting for the statistical effects of generalized cortical thinning. In light of the preponderance of female cases of hip fracture, these newly described microscopic cortical defects merit consideration regarding their origins, their possible role in weakening the femur, how they might be detected in vivo and how they might be prevented from developing prior to hip fracture.

The role of cortical bone in preserving the hip's integrity in a fall has attracted long-standing research interest [18]. It seems likely that the majority of hip fractures occur during a sideways or backwards fall with less than $5 \%$ occurring spontaneously without trauma [19, 20]. A fall onto the greater trochanter creates sudden mechanical loads that differ substantially in magnitude and direction from those imposed by walking or standing, these being the normal loads experienced by the elderly human femur [21]. In resisting these fall-related loads it is essential that the thin superior and posterior femoral neck cortices should remain intact; mechanical failure might otherwise propel a locally expanding crack into a complete hip fracture [20]. Subcortical trabeculae provide some protection [22]; but hip fracture cases have reduced trabecular as well as cortical bone in the femoral neck as shown by non-invasive imaging studies of the contra-lateral hip $[15,16,23]$.

It is not yet clear how such microscopic defects may be detected by current imaging technologies given ethical constraints on X-ray dosage; but it seems possible that the larger local defects detected in recent in vivo work either using conventional CT technology applied 
directly to the femoral neck [23] or applied to other regions of the skeleton with higher resolution CT technology and software designed to measure porosity $[24,25]$ might measure statistically associated phenomena and therefore point the way to better prediction of hip fracture. Poole and colleagues have developed new methods for analyzing in vivo computed tomography data that extract additional information from clinical images. They have found that increased femoral neck length and width are associated with the presence of focal defects in the superior region $[11,23,26]$, where the cortex is thinnest in cases and controls alike. It is not certain that the findings of Poole and colleagues directly relate to the new findings we report in this paper, if only because the cortical regions most affected are anatomically distinct. However a hip fracture involves all parts of the femoral cortex and the fracture process is likely to proceed very rapidly from one part to another over microseconds [27].

To date, most research interest in the mechanical failure mechanisms underlying hip fracture has focused on the quantitative net loss of cortical or trabecular bone in vulnerable regions such as the femoral neck or trochanter [20,23, 28]; or alternatively on submicroscopic mechanisms protecting against microscopic crack propagation and their possible degradation in aging bone tissue [29-31]. Less attention has been paid towards the effects of adverse local or microscopic distributions of the mechanical forces induced by a fall that might locally rupture the integrity of the femoral cortex. This is in contrast to the field of mechanical engineering in which interest has centred for a century on the role in initiating structural failure of small flaws in structures or materials. Potentially, mechanical loads many times higher than average in microscopic locations might initiate a crack in a hard material that expands into a macrofracture. One such mechanism that has received attention is the possible propensity to buckling of the thinned supero-lateral cortex of the elderly femoral neck [22, 32].

Our results suggest that the effects on their surrounding mechanical load distributions of the detailed geometry of unfilled cortical cavities requires further scrutiny. The regions of extreme cortical thinning we have described, are necessarily accompanied by microscopically sharp curves and corners that, as Inglis first showed a century ago [33], lead to marked increases in local stresses. Defects of circular cross section in plate-like structures increase local stresses depending on their size and other factors relating to the geometry and material properties of the surrounding material. However, any comparable defect that deviates from circularity will further increase local stress. In Inglis's example, the stretching with prolonged service life of rivet holes in ships' hulls from circular to an ellipsoid shape was shown to greatly increase surrounding stresses and have been held responsible for ships breaking up and sinking in storms. In the 
same way, it seems likely that microscopically thinned cortical regions or irregularly-shaped porosities are accompanied by locally elevated stresses when the proximal femur is loaded mechanically by a fall. Potentially, such stresses might overcome the toughening mechanisms that in normal bone prevent a microcrack expanding into a macrocrack ie a clinical fracture.

The methodology we used for determining cortical width operates by first expanding all the cavities in the cortex by $0.05 \mathrm{~mm}$ in every direction. Therefore it numerically fenestrates some trabecular or trabecular-like structures on the endosteal side of the cortex (ie those measured as $<0.1-0.14 \mathrm{~mm}$ thick) that historically were not trabecular bone, but have become trabecularised through osteoclastic activity. Enlarging cortical canals that develop over the course of time might thereby develop thin structures 100 micrometers in thickness or less separating them from the marrow space and associated trabecular bone $[3,4,34]$. Because of the way the software was written, so that it explicitly removed trabecular connections, these fenestrations are not reversed when the cortical thickness is restored during the running of the program, as shown in Fig 1.

The tendency for femoral and other cortical canals to enlarge with aging has attracted more attention with the development of improved 3-dimensional imaging [34, 35]. The possibility that this tendency to canal enlargement may be exaggerated in cases of hip fracture and that the excessive numbers of extremely thin cortical regions reflect this, links the present study with the previous work of Jordan et al [3] and Bell et al [4, 36]. They showed that in hip fracture cases remodeling osteons in femoral cortical bone tended to cluster anatomically more than in agematched controls, with the potential to merge and form enlarged composite osteons containing giant cavities of irregular shape, reducing the potential of osteoblasts to fill the space excavated with sufficient new cortical bone. An intriguing finding in the present study regarding the occurrence of these very thin cortical regions was that they clustered infero-posteriorly (ie at 270-315 degrees of arc from inferiorly). This, as van Rietbergen and Verhulp showed is on the neutral axis in relation to the loading imposed on the femoral neck in stance or slow walking; and also in the neutral axis during a fall onto the greater trochanter, which reverses the loading direction [21]. The implied lack of adequate mechanical loading in this cortical quadrant is consistent with a locally catabolic effect in the frail elderly [37].

To our knowledge, this is the first histological case-control study of male femoral neck cortical thickness in femoral neck fracture, reflecting their relative rarity compared to female cases. It is noteworthy that the relative case versus control differences in cortical thicknesses across all 
eight octants were some $40 \%$ larger in the men than in the women; whereas in contrast we found no excess of extremely thin cortical widths in the male cases. In women we could not reproduce our early finding on a small sample [2] that cortical bone loss was most severe in hip fracture cases across the supero-posterior to infero-anterior axis.

The present study has limitations. Although it contains substantial numbers of cases and controls, the requirement that the cortex should be intact resulted in a high rejection rate for cases that did not fit this criterion, in particular the cases of mid-neck fracture. Another limitation was that we had no Finnish controls so that the Finnish cases had to be compared to British controls and cases. The requirement for an intact cortex resulted in two limitations, the first being that inevitably we studied a nearby region that was some distance from the fracture surface; in turn there was relative over-representation of sub-capital fracture cases so that to generalize our conclusions to include mid-neck fractures requires the assumption that similar abnormalities existed in this more distal fracture plane. Requiring an intact cortex may also have led to the over-representation of fracture cases with less severe osteoporosis, as suggested by Bell et al and Mayhew et al [2, 38], who suggested that osteoporosis was more severe in cases with incomplete cortices. Because due to software design we could not discriminate between very thin cortical regions and regions of cortex containing large cavities that were roofed on their endosteal side by thin bridges of previously eroded cortical bone, we were unable to distinguish between these two alternative structural features; nor did we explore the possibility that some at least of the statistical determinants retrospectively associated with fracture risk were related to ongoing bone remodeling.

In conclusion we have found evidence that in addition to general thinning of femoral neck cortical bone, the structure of the femoral neck cortex in female cases of intra-capsular hip fracture is characterised by microscopic regions where thinning is extreme (cortical thickness reduced to $<0.1 \mathrm{~mm}$ ) with structural support being provided across the near cortical void by at most an endosteal bony bridge of thin trabecularised cortex. These microscopic defects might contribute to the risk of hip fracture by contributing to substantial increases in local mechanical stress during a sideways or backwards fall. This would increase the probability of complete fracture. In retrospective logistic regression modeling, the numbers of these microscopic cortical defects present in a cross section contributed perhaps more strongly than general cortical thinning towards the correct retrospective assignment in logistic regression 
analysis of case or control status. In consequence, prevention of sub-periosteal osteoclastic tunnelling has the potential to explain in part the additional benefits, beyond an increase in bone density, of treatments that reduce excessive bone resorption. Similarly, stimulation by anabolic treatments of new bone formation on previously resorbed or sub-periosteal surfaces might have a comparable strengthening effect. Further studies in women with hip fracture requiring microscopy are needed to investigate the mechanisms whereby these newly described cortical defects develop.

\section{Acknowledgements:}

This study was funded in part by: EU Biomed-1 grant BMH1CT920182; MRC Programme Grant G9321536; the 1995/6 Wellcome Trust/ Sigrid Juselius Foundation Travelling Research Fellowship grant to $\mathrm{H}$. Kröger; an American Society for Bone and Mineral Research Bridge Funding Award, 2007-8; and by the NIHR Biomedical Research Centre (OxBRC) at the Botnar Research Centre, Nuffield Orthopaedic Centre, Oxford. We thank Professor Adrian Dixon for classifying pre-operative radiographs for fracture type. 
Table 1 Characteristics of Participants Studied

\begin{tabular}{|c|c|c|c|c|c|c|}
\hline Sex & Status & Number & $\begin{array}{l}\text { Age } \\
\text { (mean) } \\
\text { years }\end{array}$ & $\begin{array}{l}\text { Age } \\
(95 \% \mathrm{CI})\end{array}$ & $\begin{array}{l}\text { Max- } \\
\text { Min } \\
\text { Ratio } \\
\text { (mean) }\end{array}$ & $\begin{array}{l}\text { Max- } \\
\text { Min } \\
\text { Ratio } \\
(95 \% \\
\text { CI) }\end{array}$ \\
\hline \multirow[t]{2}{*}{ FEMALE } & CASE & 50 & 78.6 & $74-84$ & 1.19 & $\begin{array}{l}1.05- \\
1.42\end{array}$ \\
\hline & CONTROL & 23 & 80.9 & $73-88$ & 1.26 & $\begin{array}{l}1.07- \\
1.42\end{array}$ \\
\hline \multirow[t]{2}{*}{ MALE } & CASE & 14 & 79.5 & 73-88 & 1.31 & $\begin{array}{l}1.06- \\
1.85\end{array}$ \\
\hline & CONTROL & 25 & 78.1 & $50-93$ & 1.22 & $\begin{array}{l}1.04- \\
1.50\end{array}$ \\
\hline
\end{tabular}


TABLE 2 Effects of Independent Determinants on Mean Cortical Widths (NS p >0.05)

\begin{tabular}{|l|l|l|}
\hline Determinant & Women & Men \\
\hline Age & $-6.7 \%$ per decade & NS \\
\hline Fracture status (fracture) & $-13 \%$ & $-32 \%$ \\
\hline $\begin{array}{l}\text { Max:Min Ratio (per 10\% } \\
\text { increase) }\end{array}$ & $+14 \%$ & NS \\
\hline
\end{tabular}


Table 3 Characteristics of hip fracture cases compared to controls: analysis by cortical octants.

\section{WOMEN}

Level geometric mean widths geometric mean widths $\%$

reduction cases

Cases Controls

Anterior $\quad 0.72$

1.10

$35 \%$

Inf-Ant 1.80

2.67

$32 \%$

Inf-Post $\quad 0.83$

1.73

$52 \%$

Inferior $\quad 2.49$

3.31

$25 \%$

Posterior $\quad 0.53$

0.82

$35 \%$

Sup-Ant $\quad 0.60$

0.76

$21 \%$

Sup-Post $\quad 0.61$

0.75

$19 \%$

Superior $\quad 0.55$

0.72

$23 \%$

\section{MEN}

Anterior

0.60

1.25

$52 \%$

Inf-Ant 1.37

2.86

$52 \%$

Inf-Post $\quad 0.23$

1.80

$87 \%$

Inferior $\quad 1.26$

3.42

$63 \%$

Posterior $\quad 0.24$

0.88

$72 \%$

Sup-Ant $\quad 0.58$

0.85

$31 \%$

Sup-Post $\quad 0.55$

0.83

$34 \%$

Superior $\quad 0.52$

0.76

$32 \%$ 
Table 4 Variability of Cortical widths by cortical octants in cases and controls: values approaching 1 indicate low variability in cortical width across the 9 measurements per octant; values closer to zero indicate higher variability of cortical width, with increased proportions of relatively

low values.

\begin{tabular}{|c|c|c|c|c|c|}
\hline \multirow{2}{*}{$\begin{array}{l}\text { Angle } \\
\text { relative to } \\
\text { Inferior } \\
\text { (degrees) }\end{array}$} & \multirow[t]{2}{*}{$\begin{array}{l}\text { Octant } \\
\text { name }\end{array}$} & \multicolumn{2}{|c|}{$\begin{array}{l}\text { Ratios for Median*: } \\
\text { Minimum CW to mean CW }\end{array}$} & \multicolumn{2}{|c|}{$\begin{array}{l}\text { Ratios for } 10^{\text {th }} \text { Centile**: } \\
\text { Minimum CW to mean CW }\end{array}$} \\
\hline & & Controls $n=48$ & Cases $n=64$ & Controls $n=48$ & Cases $n=64$ \\
\hline $0^{\circ}$ & Inferior & 0.72 & 0.69 & 0.55 & 0.33 \\
\hline $45^{\circ}$ & $\begin{array}{l}\text { Infero- } \\
\text { anterior }\end{array}$ & 0.65 & 0.60 & 0.46 & 0.20 \\
\hline $90^{\circ}$ & Anterior & 0.58 & 0.47 & 0.25 & 0.14 \\
\hline $135^{\circ}$ & $\begin{array}{l}\text { Supero- } \\
\text { Anterior }\end{array}$ & 0.46 & 0.48 & 0.19 & 0.17 \\
\hline $180^{\circ}$ & Superior & 0.45 & 0.46 & 0.18 & 0.10 \\
\hline $225^{\circ}$ & $\begin{array}{l}\text { Supero- } \\
\text { posterior }\end{array}$ & 0.53 & 0.55 & 0.21 & 0.22 \\
\hline $270^{\circ}$ & Posterior & 0.51 & 0.53 & 0.25 & 0.14 \\
\hline $315^{\circ}$ & $\begin{array}{l}\text { Infero- } \\
\text { posterior }\end{array}$ & 0.69 & 0.56 & 0.45 & 0.12 \\
\hline
\end{tabular}

* The median was calculated as the median of the ratios of minimum CW: mean CW in each octant for each participant. The group median values shown were then calculated for all controls and all cases combining men and women; $\mathrm{n}=9 \mathrm{CW}$ measurements for each octant per participant. $* *$ From the same ratios, the group $10^{\text {th }}$ centile (quantile) value was also calculated and expressed as a ratio to the mean $\mathrm{CW}$. 
FIGURES

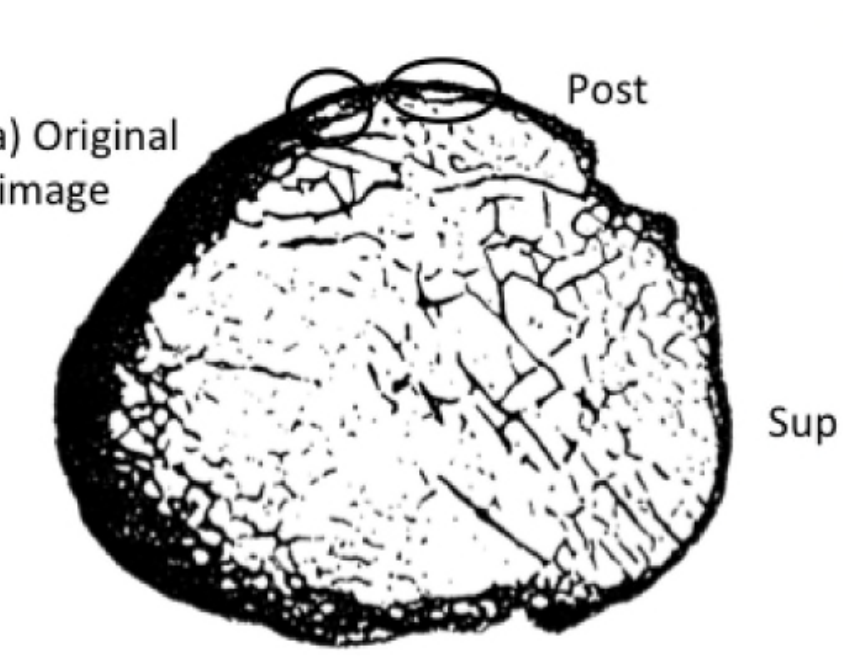

b) Invert

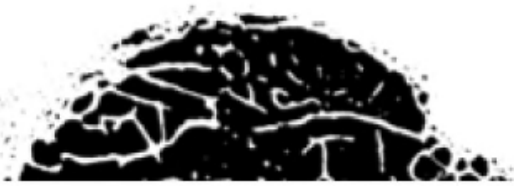

c) Dilate

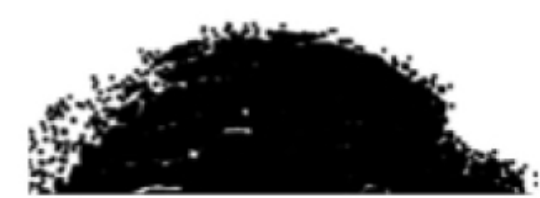

d) Fill marrow cavity

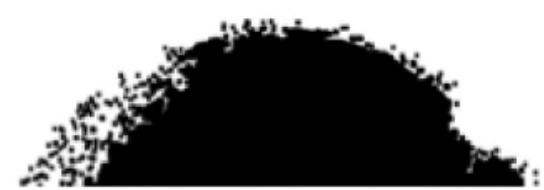

e) Clear cortical

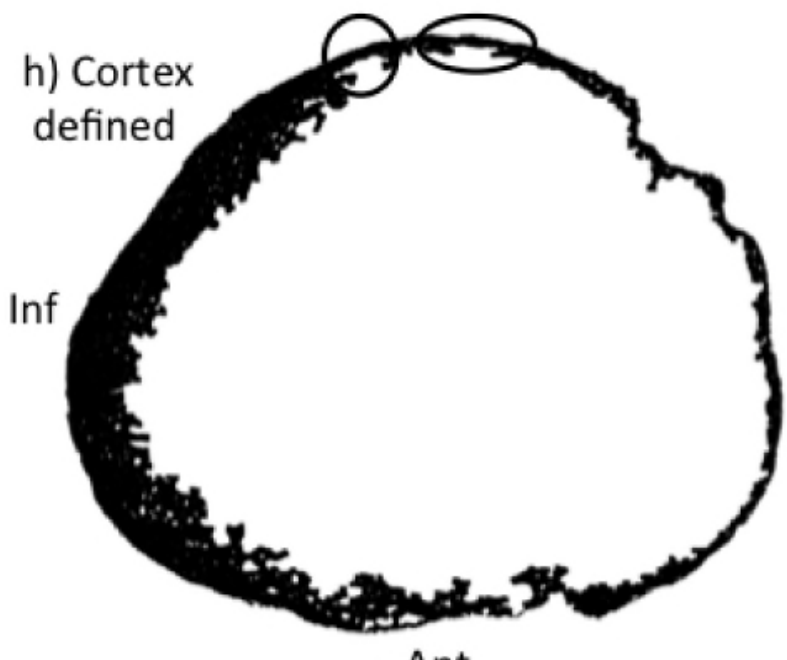
canals

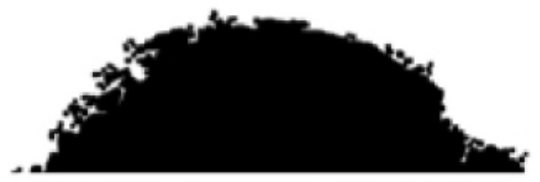

f) Erode back marrow cavity

g) Trace endosteal boundary

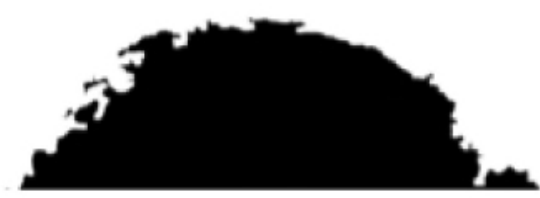

Fig 1

Ant 


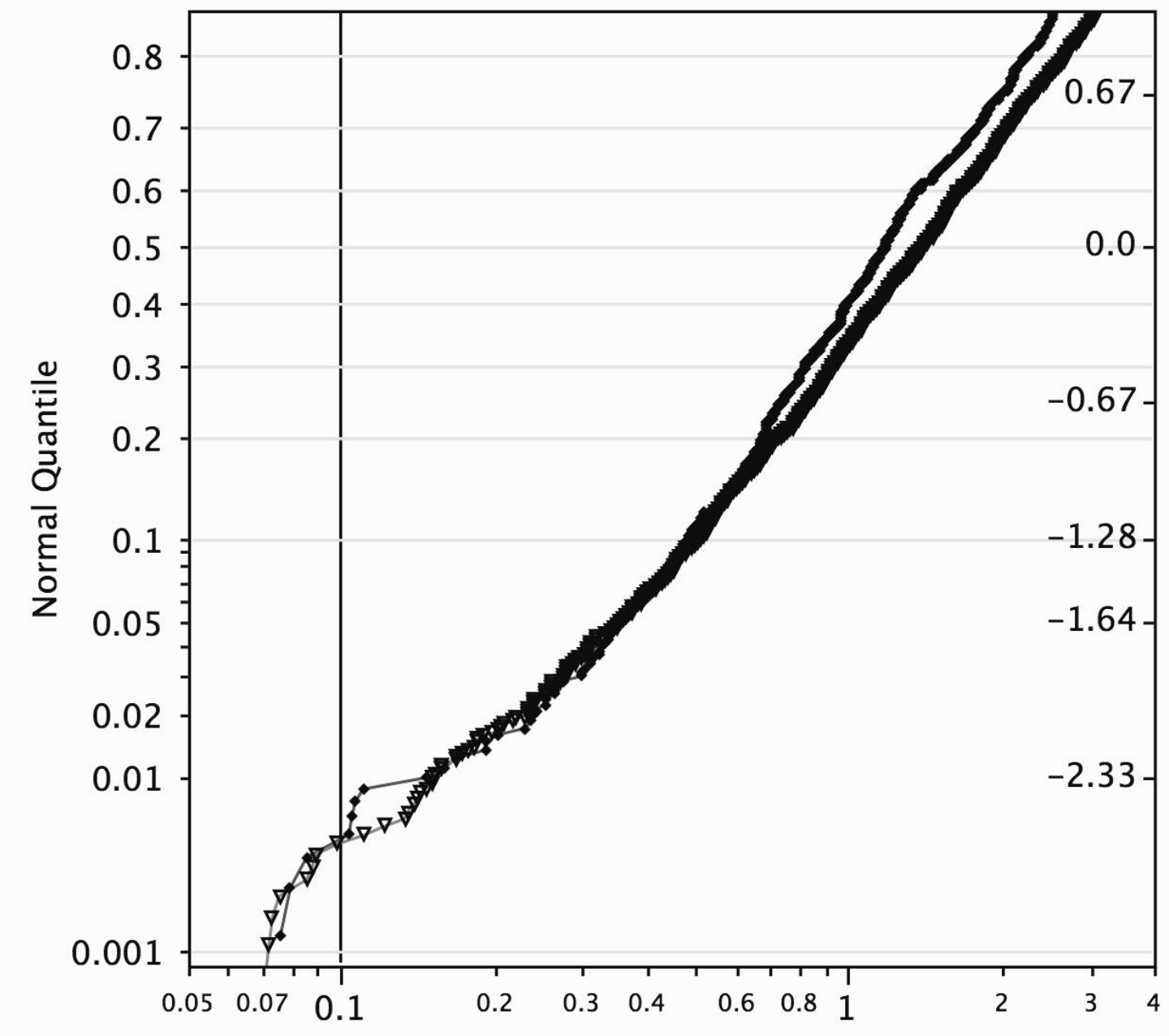

Fig 2a

Cortical Width (mm) 


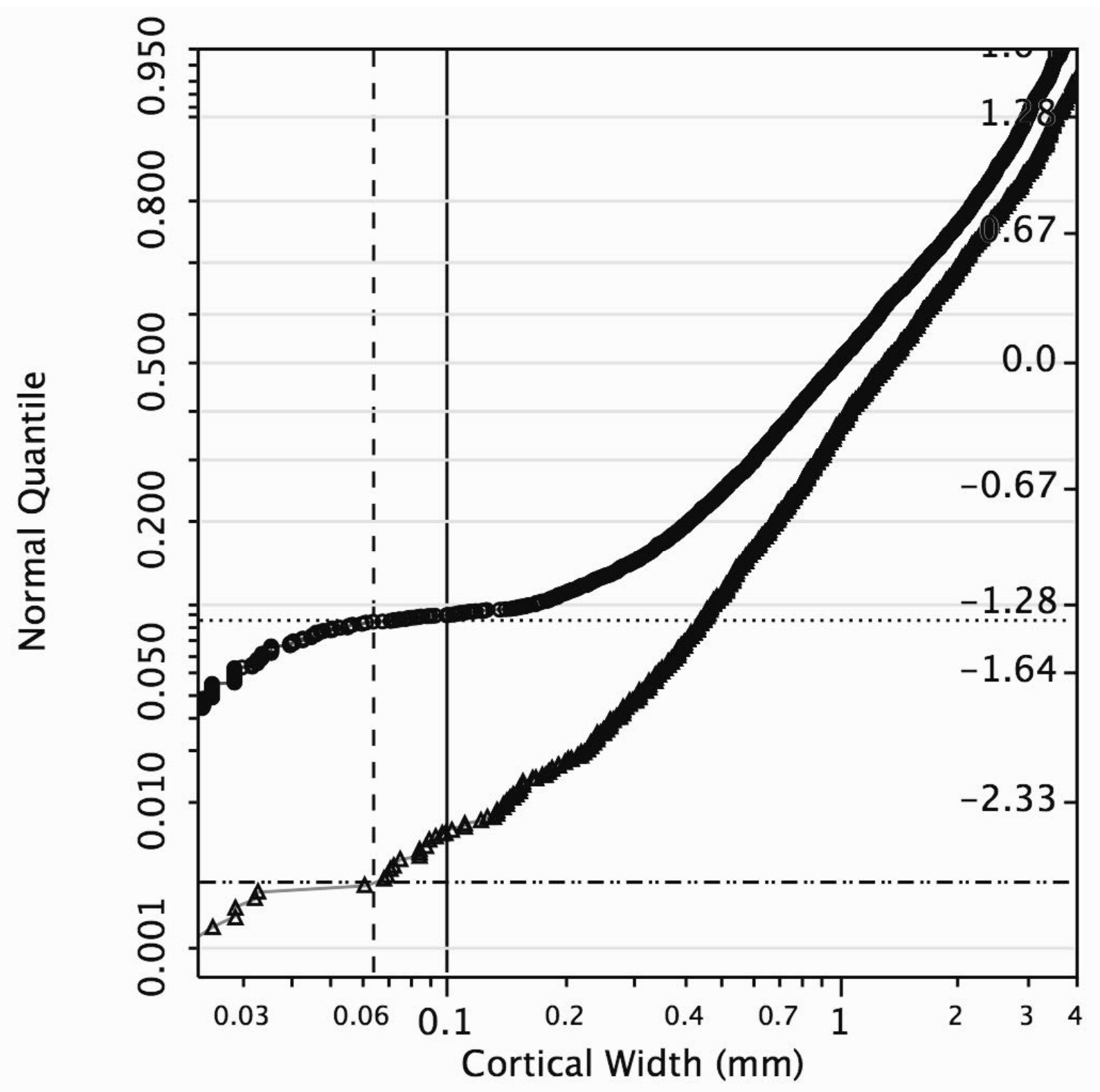

Fig 2b 


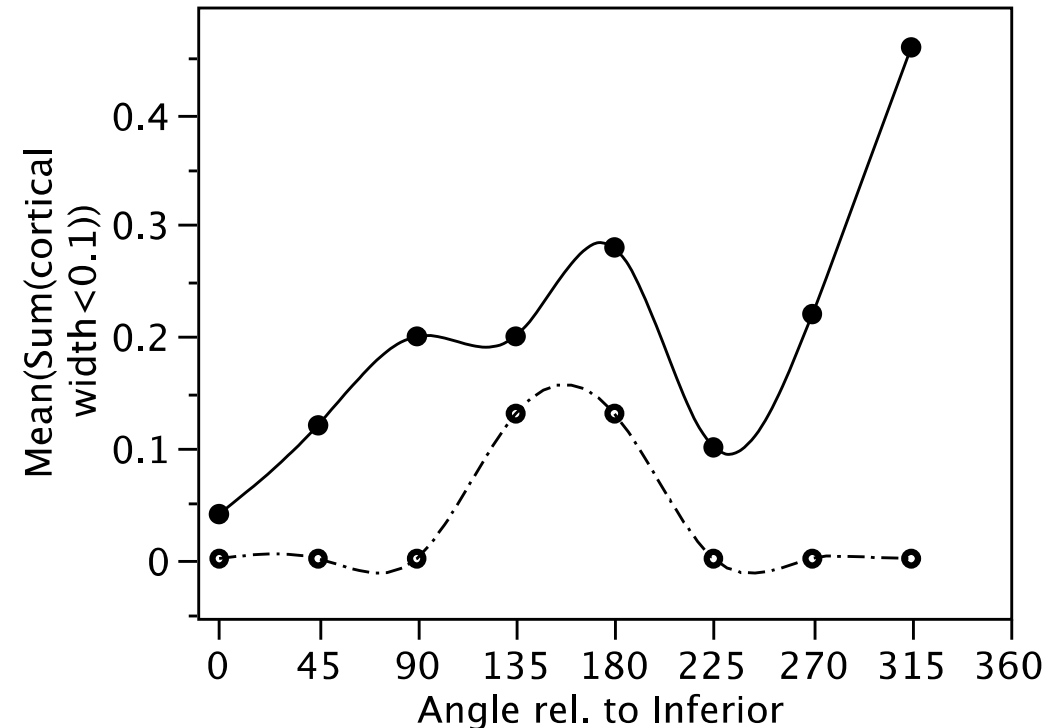

Fig 3 


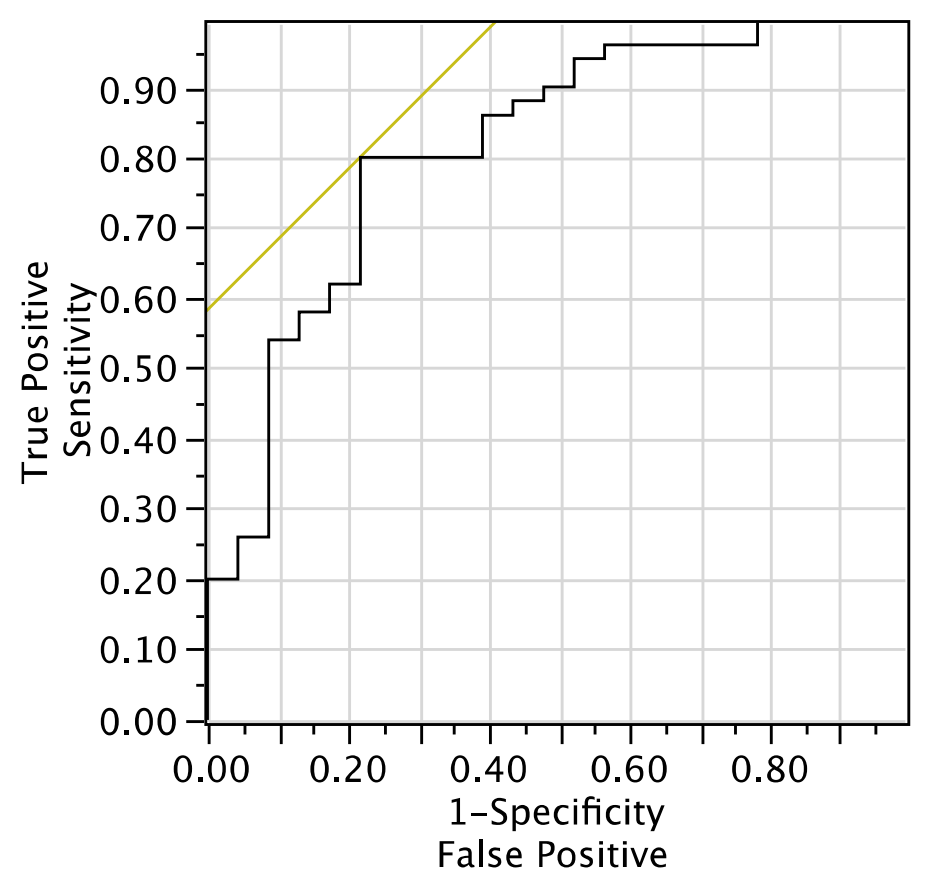

Women

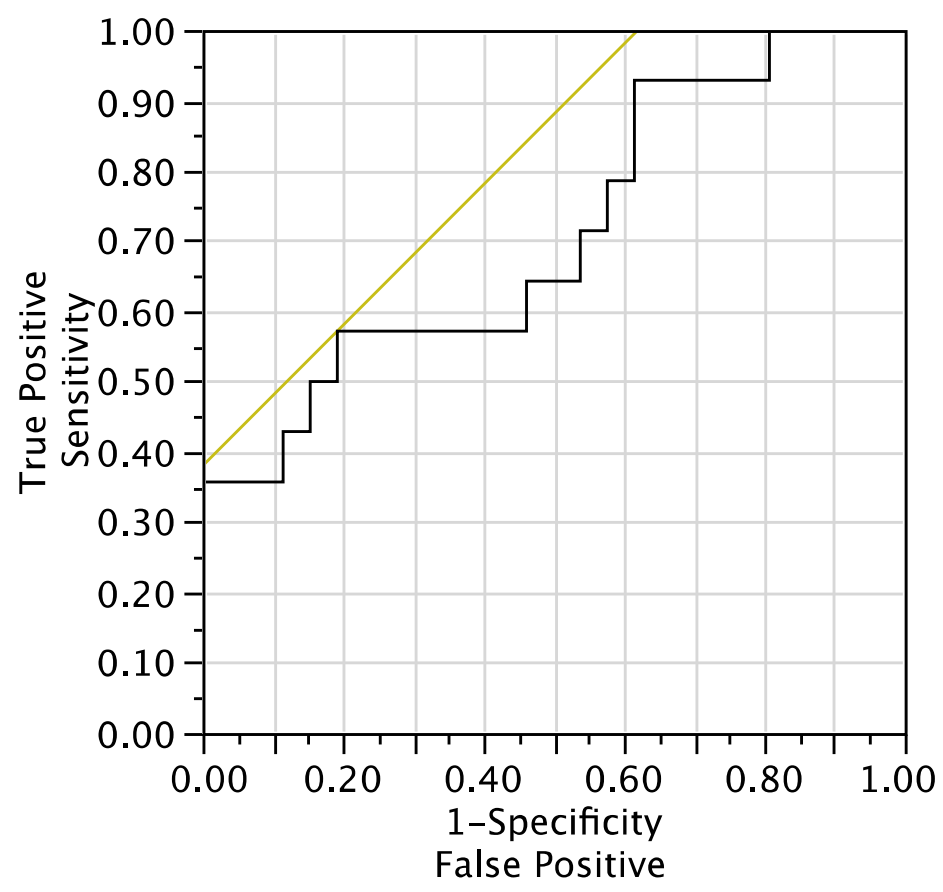

Men 


\section{FIGURE CAPTIONS}

Fig 1: Image at top left (a) shows the captured image of one femoral neck cross section. Top right: Only the posterior portion of the cortex and underlying trabeculae are shown to illustrate the following six stages of processing. First (b) the image is inverted prior to being dilated (c: see text). The marrow cavity (d) is cleared of bony elements ("filled") and the remaining cortical canals are also filled (e). Next the marrow cavity is eroded back by the same number of pixels by which it was originally expanded (f). The endosteal boundary is then traced (g) and (h: bottom left) the defined cortex is displayed. Two rings are traced in super-position on (a) and (h) to illustrate two large possibly cortical canals located posteriorly; one ring is circular and the other oval in shape. It will be seen that endosteally both these apparent canals are closed by sectors of bone that have been defined as trabecular, so that neither is included in the cortex as defined by our algorithm. Smaller canals within thicker segments of cortex remain included in the cortical bone and therefore have no effect on measurements of cortical width.

Fig 2 M \& Fig 2F. Middle and lower portions of Q-Q plots of valid cortical width measurements in all octants, cases (dots) and controls (inverted triangles). Males (M) and females $(F)$ are shown separately The deviation of their $Q-Q$ plots from the $Q-Q$ plot of the controls is only seen in the female cases and is most marked in the range $0.05-0.125 \mathrm{~mm}$.

Fig 3 Plot of mean numbers of CWs $<0.1 \mathrm{~mm}$ (maximum 9) as a function of angular distance relative to inferior in female cases (continuous line, solid symbols) and controls (dashed line, open symbols). The lines are empirical best-fit spline functions. 0 degrees $=$ inferior; $45=$ infero-anterior; 90 = anterior; 135 = supero-anterior; 180 = superior; 225 = supero-posterior; 270 = posterior; 315 = infero-posterior.

Fig 4 ROC curves showing plots of sensitivity vs 1-specificity in women and men respectively for logistic models retrospectively "predicting" hip fracture from data that included log transformed mean CW and numbers of individual CW measurements $0.1 \mathrm{~mm}$ or lower, adjusted for age and the max:min ratio (see text). 


\section{REFERENCES}

[1]. Bell, K. L., Loveridge, N., Power, J., Stanton, M., Meggitt, B. F., and Reeve, J. Regional differences in cortical porosity in the fractured femoral neck. Bone 24:57$64 ; 1999$.

[2]. Bell, K., Loveridge, N., Power, J., Garrahan, N., Stanton, M., Lunt, M., Meggitt, B., and Reeve, J. Structure of the femoral neck in hip fracture: cortical bone loss in the inferoanterior to superoposterior axis. Journal of Bone and Mineral Research $14: 112-120 ; 1999$.

[3]. Jordan, G., Loveridge, N., Bell, K. L., Power, J., Rushton, N., and Reeve, J. Spatial clustering of osteonal remodelling: a cause of focal weakness in the femoral neck cortex in hip fracture. Bone 26:305-313; 2000.

[4]. Bell, K. L., Loveridge, N., Jordan, G. R., Power, J., Constant, C. R., and Reeve, J. A novel mechanism for the induction of the increased cortical porosity in cases of intracapsular hip fracture. Bone 27:297-304; 2000.

[5]. Crabtree, N., Loveridge, N., Parker, M., Rushton, N., Power, J., Bell, K. L., Beck, T. J., and Reeve, J. Intracapsular hip fracture and the region-specific loss of cortical bone: analysis by Quantitative Computed Tomography (pQCT). Journal of Bone \& Mineral Research 16:1318-1328; 2001.

[6]. Bell, K., Loveridge, N., Power, J., Rushton, N., and Reeve, J. Intracapsular hip fracture: Increased cortical remodelling in the thinned and porous anterior region of the femoral neck. Osteoporosis International 10:248-257; 1999.

[7]. Moyes, S. T., and Wand, J. S. A new procedure for biopsy of the femoral neck in the study of Type 2 osteoporosis. Bone 14:183; 1993.

[8]. Caballero, A., Loveridge, N., Pitsillides, A., Parker, M., Kaptoge, S., Lyon, A., and Reeve, J. Osteocytic expression of constitutive NO synthase isoforms in the femoral neck cortex: a case-control study of intra-capsular hip fracture. Journal of Bone \& Mineral Research 20:268-273; 2005.

[9]. Nevitt, M. C., Johnell, O., Black, D. M., Ensrud, K., Genant, H. K., Cummings, S. R., and for the Study of Osteoporotic Fractures Research Group Bone Mineral Density Predicts Non-spine Fractures in Very Elderly Women. Osteoporosis International 4:325-331; 1994.

[10]. Cummings, S. R., Black, D. M., Nevitt, M. C., Browner, W., Cauley, J., Ensrud, K., Genant, H. K., Palermo, L., Scott, J., and Vogt, T. M. Bone Density at Various Sites for Prediction of Hip Fractures. Lancet 341:72-75; 1993.

[11]. Poole, K. E. S., Treece, G. M., Mayhew, P. M., Vaculík, J., Dungl, P., Horák, M., Štěpán, J. J., and Gee, A. H. Cortical thickness mapping to identify focal osteoporosis in patients with hip fracture. PLoS One 7:e38466 Epub 2012 Jun 11; 2012.

[12]. Carpenter, R. D., Beaupré, G. S., Lang, T. F., Orwoll, E. S., Carter, D. R., and Osteoporotic Fractures in Men (MrOS) Study Group New QCT analysis approach shows the importance of fall orientation on femoral neck strength. J Bone Miner Res. 20:1533-1542; 2005.

[13]. Black, D. M., Bouxsein, M. L., Marshall, L. M., Cummings, S. R., Lang, T. F., Cauley, J. A., Ensrud, K. E., Nielson, C. M., Orwoll, E. S., and Osteoporotic Fractures in Men (MrOS) Research Group Proximal femoral structure and the prediction of hip fracture in men: a large prospective study using QCT. J Bone Miner Res. 23:1326-1333; 2008.

[14]. Kaptoge, S., Beck, T. J., Reeve, J., Stone, K. L., Hillier, T. A., Cauley, J. A., and Cummings, S. R. Prediction of incident hip fracture risk by femur geometry variables measured by hip structural analysis in the Study of Osteoporotic Fractures. Journal of Bone \& Mineral Research 23:1892-1904; 2008.

[15]. Johannesdottir, F., Poole, K. E. S., Reeve, J., Siggeirsdottir, K., Aspelund, T., Mogensen, B., Jonsson, B. Y., Sigurdsson, S., Harris, T. B., Gudnason, V. G., and Sigurdsson, $G$. Distribution of cortical bone in the femoral neck and hip fracture: $A$ 
prospective case-control analysis of 143 incident hip fractures; the AGESREYKJAVIK Study. Bone 48(6):1268-76:1268-1276; 2011.

[16]. Johannesdottir, F., Aspelund, T., Reeve, J., Poole, K. E. S., Sigurdsson, S., Harris, T. B., Gudnason, V. G., and Sigurdsson, G. Age-related Regional Losses of Cortical and Trabecular Bone in Femoral Neck in elderly Women and Men: The AGESReykjavik Longitudinal Study. Journal of Bone \& Mineral Research 28:2165-2176; 2013.

[17]. Treece, G. M., Poole, K. E. S., and Gee, A. H. Imaging the femoral cortex: thickness, density and mass from clinical CT. Med Image Anal. 16:952-965; 2012.

[18]. Reeve, J. Role of cortical bone in hip fracture. Bonekey Rep..Jan 13;6:867. doi: 10.1038/bonekey.2016.82; 2017.

[19]. Nevitt, M. C., and Cummings, S. R. Type of fall and risk of hip and wrist fractures: the study of osteoporotic fractures. The Study of Osteoporotic Fractures Research Group. Journal of the American Geriatric Society 42:797-798; 1993.

[20]. Reeve, J., and Loveridge, N. The Fragile Elderly Hip: Mechanisms Associated with Age-Related Loss of Strength and Toughness. Bone 61:138-148; 2014.

[21]. Verhulp, E., van Rietbergen, B., and Huiskes, R. Load distribution in the healthy and osteoporotic human proximal femur durng a fall to the side. Bone 42:30-35; 2008.

[22]. Thomas, C. D. L., Mayhew, P. M., Power, J., Poole, K. E. S., Loveridge, N., Clement, J. G., Burgoyne, C. J., and Reeve, J. Femoral Neck Trabecular Bone: Loss with Ageing and Role in Preventing Fracture. J Bone and Miner Res 24:1808-18; 2009.

[23]. Poole, K. E., Skingle, L., Gee, A., H, Turmezei, T. D., Johannesdottir, F., Blesic K, Rose, C., Vindlacheruvu, M., Donell, S., Vaculik, J., Dungl, P., Horak, M., Stepan, J. J., Reeve, J., and Treece, G. M. Focal osteoporosis defects play a key role in hip fracture. Bone 94:124-134; 2017.

[24]. Bala, Y., Zebaze, R., Ghasem-Zadeh, A., Atkinson, E. J., luliano, S., Peterson, J. M., Amin, S., Bjørnerem, Å., Melton 3rd, L. J., Johansson, H., Kanis, J. A., Khosla, S., and Seeman, E. Cortical porosity identifies women with osteopenia at increased risk for forearm fractures. J Bone Miner Res. 29:1356-1362; 2014.

[25]. Ahmed, L. A., Shigdel, R., Joakimsen, R. M., Eldevik, O. P., Eriksen, E. F., GhasemZadeh, A., Bala, Y., Zebaze, R., Seeman, E., and Bjørnerem, Å. Measurement of cortical porosity of the proximal femur improves identification of women with nonvertebral fragility fractures. Osteoporos Int. 26:2137-2146; 2015.

[26]. Gee, A. H., Treece, G. M., Tonkin, C. J., Black, D. M., and Poole, K. E. S. Association between femur size and a focal defect of the superior femoral neck. . Bone 81:60-66; 2015.

[27]. de Bakker, P. M., Manske, S. L., Ebacher, V., Oxland, T. R., Cripton, P. A., and Guy, $P$. During sideways falls proximal femur fractures initiate in the superolateral cortex: evidence from high-speed video of ex vivo fractures. J Biomechanics 42:1917-1925; 2009.

[28]. Zysset, P. K. Where does hip fracture initiate? Bonekey Rep. ; 2014 Dec 3;3:601. doi: 10.1038/bonekey.2014.96. eCollection 2014.

[29]. Busse, B., Djonic, D., Milanovic, P., Hahn, M., Puschel, K., Ritchie, R. O., Djuric, M., and Amling, M. Decrease in the osteocyte lacunar density accompanied by hypermineralized lacunar occlusion reveals failure and delay of remodelling in aged human bone. Aging Cell 9:1065-1075; 2010.

[30]. Launey, M. E., Buehler, M. J., and Ritchie, R. O. On the mechanistic origins of toughness in bone. Annual Review of Materials Research 40:25-53; 2010.

[31]. Zimmermann, E. A., Busse, B., and Ritchie, R. O. The fracture mechanics of human bone: influence of disease and treatment. Bonekey Rep. 4; 2015 Sep 2.

[32]. Mayhew, P. M., Thomas, C. D. L., Clement, J. G., Loveridge, N., Beck, T. J., Bonfield, W., Burgoyne, C. J., and Reeve, J. Relation between age, femoral neck cortical stability, and hip fracture risk. Lancet 366:129-135; 2005. 
[33]. Inglis, C. E. Stress in a plate due to the presence of sharp corners and cracks. Transactions of the Royal Institute of Naval Architects 60:219-241; 1913.

[34]. Cooper, D. M., Thomas, C. D., Clement, J. G., Turinsky, A. L., Sensen, C. W., and Hallgrimsson, B. Age-dependent change in the 3D structure of cortical porosity at the human femoral midshaft. Bone 40:957-965; 2007.

[35]. Cooper, D. M. L., Erickson, B., Peele, A. G., Hannah, K., Thomas, C. D., and Clement, J. G. Visualization of 3D osteon morphology by synchrotron radiation micro-CT. J Anat. 219:481-489; 2011.

[36]. Bell, K. L., Loveridge, N., Reeve, J., Thomas, C. D. L., Feik, S. A., and Clement, J. G. Super-osteons (remodelling clusters) in the cortex of the femoral shaft: influence of age and gender. Anatomical Record 264:378-386; 2001.

[37]. Meakin, L. B., Price, J. S., and Lanyon, L. E. The Contribution of Experimental in vivo Models to Understanding the Mechanisms of Adaptation to Mechanical Loading in Bone. Front Endocrinol (Lausanne). Oct 1;5:154. doi: 10.3389/fendo.2014.00154. eCollection 2014; 2014.

[38]. Mayhew, P., Kaptoge, S., Loveridge, N., Power, J., Kroger, H. P. J., Parker, M., and Reeve, J. Discrimination between cases of hip fracture and controls is improved by hip structural analysis compared to areal bone mineral density. An ex vivo study of the femoral neck. Bone 34:352-361; 2004. 